\title{
Misdiagnosis of multiple synchronous small bowel adenocarcinomas as intestinal tuberculosis: a case report
}

\author{
Qiwei $\mathrm{Li}^{1 \dagger}$, Tao Chen ${ }^{1 \dagger}$, Hexi Cuii ${ }^{2 \dagger}$, Xiao Xiao ${ }^{3}$, Chunqiu Chen ${ }^{4}$, Zhenyu Shen ${ }^{4}, \mathrm{Fu} \mathrm{Ji}^{5^{*}}$ and Lu Yin ${ }^{4^{*}}$
}

\begin{abstract}
Background: Small bowel adenocarcinoma (SBA) is a rare malignancy that primarily occurs in the duodenum. Multiple synchronous SBA is unique rare and difficult to diagnose due to non-specific disease presentation. Protocols to identify multiple synchronous SBA during early disease stages are urgently required.

Case presentation: An elderly man experienced left lower abdominal pain and melena for 3 months. Abdominal CT showed thickening of the multiple segmental small intestinal walls. As the patient had pulmonary tuberculosis simultaneously, he was misdiagnosis as intestinal tuberculosis and received anti-spasm therapy. The treatment delayed radical resection surgery and the patient underwent palliative segmental resection of the jejunum after 4 months due to intestinal obstruction. Resected specimens showed multiple synchronous SBA (five tumors). The patient accepted chemotherapy postoperatively. Six months postoperatively, the patient died of brain metastasis.

Conclusions: We highlight how multiple synchronous SBA is rare and easily misdiagnosed. We should rule out multiple synchronous SBA when diagnosing intestinal diseases (e.g. inflammatory bowel disease, IBS). Intestinal tuberculosis may also be one of the risk factors for multiple synchronous SBA. High-risk patients should be assessed for known tumor makers, and receive gastroscopy, enteroscopy or capsule endoscopy. Doctors should obtain the pathology under endoscopy to the greatest possible degree. For suspected patients, laparotomy should be performed.
\end{abstract}

Keywords: Small bowel cancer, Small bowel adenocarcinoma, Intestinal tuberculosis, Diagnosis, High-risk

\section{Background}

Small bowel cancer is a rare malignancy that comprises less than $5 \%$ of all gastrointestinal malignancies [1]. Its annual incidence is approximately 13.9 cases per million individuals. Small bowel cancer has four common histological types: adenocarcinoma (30-40\%), carcinoid tumor (35-42\%), lymphoma (15-20\%), and sarcoma

\footnotetext{
*Correspondence: dr_jifu@163.com; yinlumastet0105@126.com

${ }^{\dagger}$ Qiwei Li, Tao Chen and Hexi Cui contributed equally to this work.

${ }^{5}$ Department of Gastrointestinal Surgery, Renji Hospital, School of Medicine, Shanghai Jiao Tong University, Shanghai 200127, China

${ }^{4}$ Department of Abdominal Surgery, Shanghai Tenth People's Hospital, Tongji University School of Medicine, Shanghai 200072, China

Full list of author information is available at the end of the article
}

(10-15\%) [2]. Small bowel adenocarcinoma (SBA) is most commonly located in the duodenum (57\%), $29 \%$ of cases are located in the jejunum, and $10 \%$ of cases are in the ileum [3]. The clinical presentation of SBA is nonspecific abdominal discomfort, including abdominal pain, nausea, vomiting, gastrointestinal bleeding and intestinal obstruction. This leads to an average delay of 6-10 months in diagnosis [4].

The majority of SBA are single with multiple synchronous SBA rare. Due this rarity, multiple synchronous SBA is frequently misdiagnosed and knowledge on the clinical characteristics, treatment modalities, and prognosis of patients is sparse, particularly in the Asian community.

(c) The Author(s). 2020 Open Access This article is licensed under a Creative Commons Attribution 4.0 International License, which permits use, sharing, adaptation, distribution and reproduction in any medium or format, as long as you give appropriate credit to the original author(s) and the source, provide a link to the Creative Commons licence, and indicate if changes were made. The images or other third party material in this article are included in the article's Creative Commons licence, unless indicated otherwise in a credit line to the material. If material is not included in the article's Creative Commons licence and your intended use is not permitted by statutory regulation or exceeds the permitted use, you will need to obtain permission directly from the copyright holder. To view a copy of this licence, visit http://creativecommons.org/licenses/by/4.0/ The Creative Commons Public Domain Dedication waiver (http://creativecommons.org/publicdomain/zero/1.0/) applies to the data made available in this article, unless otherwise stated in a credit line to the data. 
In this study, we report a case of multiple synchronous SBA in the jejunum in a 70-year-old man. The patient was misdiagnosed as intestinal tuberculosis and accepted anti-tuberculosis treatment until bowel obstruction with lymph node metastasis was observed. Palliative segmental bowel resection was performed. The patient died postoperatively after 6 months. This highlights how multiple synchronous SBA is rare and easily misdiagnosed during early stages.

\section{Case presentation}

The patient was a 70-year-old male who had experienced left lower abdominal pain and melena for 3 months. Physical examination revealed a soft abdomen with tenderness in the left lower quadrant. A mass of 5 $\mathrm{cm}$ in diameter was identified. The mass boundary was unclear and the mass was immobile. The patient had no family history. Laboratory tests included fecal occult blood ++; hemoglobin $60 \mathrm{~g} /$ liter; interferon-gamma release assays for Mycobacterium tuberculosis (TB-IGRA) +; T spot +; sputum culture: no pathogenic bacteria growth; sputum smear: no acid-fast bacilli; antituberculosis antibodies IgG -; anti-tuberculosis antibodies IgM -; erythrocyte sedimentation rate (ESR) 50 $\mathrm{mm} / \mathrm{h}$; Ca211: $12.4 \mathrm{ng} / \mathrm{ml}, \mathrm{Ca} 125$ and Ca153 were normal. SCC: $5.3 \mathrm{ng} / \mathrm{ml}$; anti-streptolysin (ASO): $133 \mathrm{IU} / \mathrm{ml}$; rheumatoid factor (RF): $17 \mathrm{IU} / \mathrm{ml}$. Pulmonary CT (Fig. 1a-b) identified a cavity lesion in the posterior segment of the upper lobe of the left lung, enlarged lymph nodes in the left hilar and mediastinum, emphysema, multiple pulmonary bullae, and posterior segmental tuberculosis. Abdominal CT identified uneven thickening of the small intestine with localized dilatation in the left middle abdomen and multiple retroperitoneal lymph nodes. Gastroscopy showed no abnormalities, but colonoscopy revealed multiple polyps in colon and rectum.
Initially, the patient was diagnosed with secondary pulmonary tuberculosis and intestinal tuberculosis in the infectious disease hospital. After 2 weeks of HERZ treatment, the abdominal pain did not alleviate.

One month later, the patient came to our hospital. Abdominal CT (Fig. 2a-b) showed multiple thickened small intestinal walls in the left abdomen and peripheral exudation. Multiple enlarged lymph nodes were identified in the surrounding and posterior peritoneum, some of which were fused. Due to the increasing levels of tumor makers (Ca125: 37.67 U/ml; Ca153: 129.5 U/ml; Ca211: $25.1 \mathrm{ng} / \mathrm{ml}$ ) and CT examinations, an intestinal tumor could not be discounted. He underwent palliative segmental resection of the jejunum. At laparotomy, five mass were identified at the jejunum $(15,30,50,70$ and $80 \mathrm{~cm}$ from the ligament of Treitz). The largest diameter was $5 \mathrm{~cm}$. The small bowel mass was of the infiltrating stenosis type and invaded the serosa. Multiple enlarged lymph nodes observed in mesentery and blood vessel roots had fused into a mass. There was no evidence of intraperitoneal dissemination or parenteral metastasis.

Pathology (No.18-01519) revealed 5 poorly differentiated small bowel carcinomas; the size of which were $3 \times$ $3 \times 1.5 \mathrm{~cm}, 6 \times 4 \times 1.5 \mathrm{~cm}, 6 \times 5 \times 1.5 \mathrm{~cm}, 6 \times 5 \times 1.5 \mathrm{~cm}$ and $5 \times 5 \times 1.5 \mathrm{~cm}$ respectively. SBA invaded the intestinal serosa. The lymph nodes $(8 / 11)$ were metastatic and the margins were negative. Tumor staging was T4N2M0 (IIIB). Immunohistochemistry: CK(+), CK7(+), CK20(-), Vimentin (+), S-100(-), MelanA (-), CD34(-), CD117(-), Dog-1 (-), ki67 positive rate 90\%, AFP (+/-), NKX3.1 (-), p40 (-), CgA (-), HER-2 (2+), MPO (-), LCA (-), cdx-2 (-), NapsinA (-), TTF-1 (-). The patient began chemotherapy postoperatively for 1 month and accepted 4 cycles of treatment (Irinotecan + Teggio). Postoperation pulmonary tuberculosis was diagnosed from positive sputum smear tests and anti-spasm
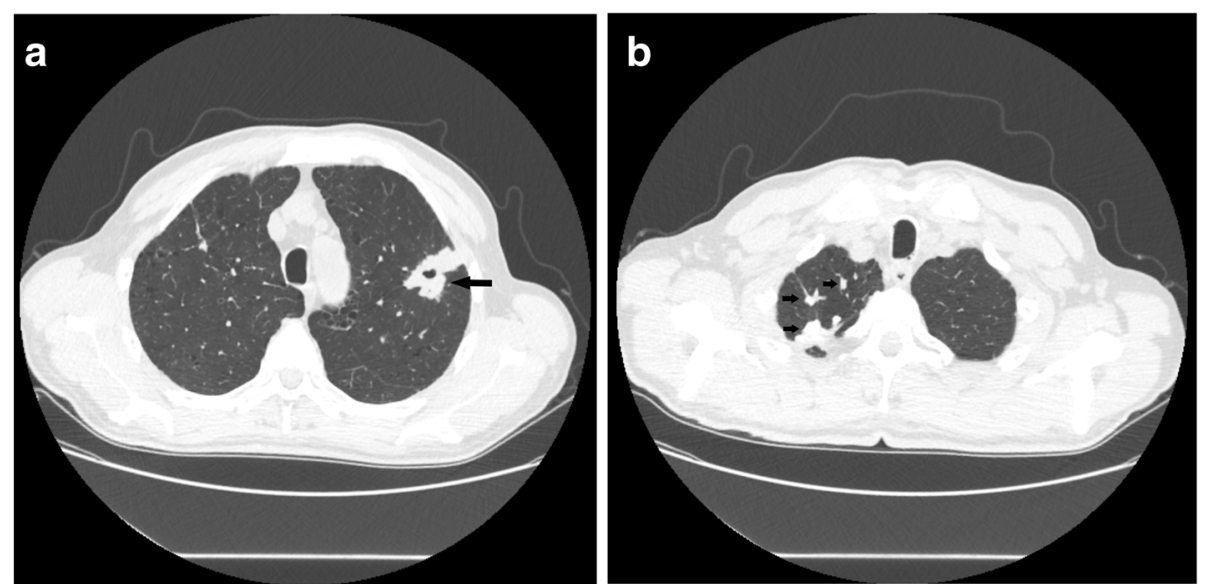

Fig. 1 Pulmonary CT. a A cavity lesion (black arrow) in the posterior segment of the upper lobe of the left lung. b Multiple nodules and spots (black arrow) in the upper lobe of the right lung 

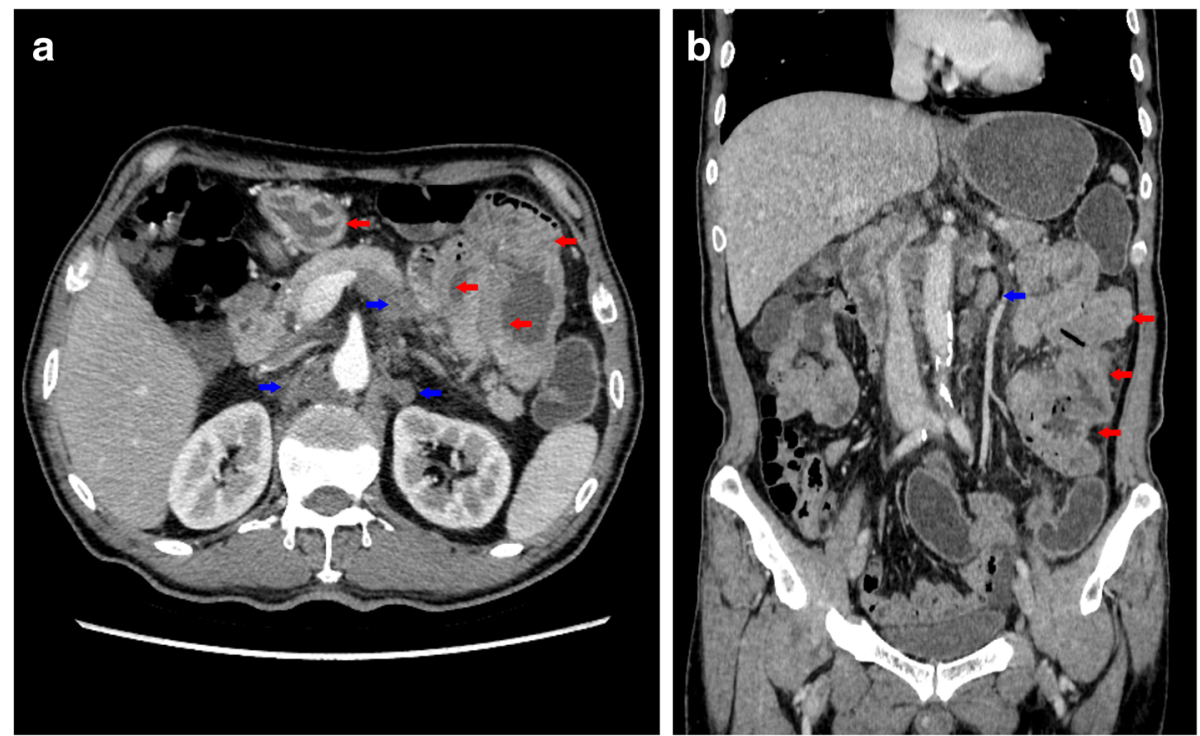

Fig. 2 Abdominal CT. a-b Multiple thickened small intestinal walls (red arrow) in the left abdomen and multiple enlarged lymph nodes (blue arrow) in the surrounding and posterior peritoneum

treatment was continued. Finally, the patient was diagnosed with multiple synchronous SBA and pulmonary tuberculosis. Six months later, the patient died from brain metastasis.

\section{Discussion and conclusions}

SBA is rare and most tumors are single and located in the duodenum. SBA often develops with vague and nonspecific gastrointestinal symptoms, including obscure bleeding, abdominal pain, nausea and vomiting, weight loss, diarrhea, and intestinal obstruction. As a result of relative infrequency and lack of clear symptoms, the diagnosis of SBA occurs at advanced stages; with $-40 \%$ of patients showing lymph node metastasis (stage III), and 35 to $40 \%$ with distant metastasis (stage IV) [5]. Multiple synchronous SBA is a unique type of SBA, with studies on these tumors sparse. Only few reports show that it is difficult to diagnose [6]. Whilst CT scans can detect the lesions, they only identify the thickening of multiple segmental small intestinal walls. This makes it difficult to distinguish multiple synchronous SBA from IBS. Our patient showed pulmonary tuberculosis and so intestinal tuberculosis was considered and the operation was delayed. Protocols to identify multiple synchronous SBA at an early stage are currently lacking and are urgently required.

Screening high-risk patients with typical symptoms is an option for early disease identification [7]. Chronic inflammation is associated with the formation of several malignant tumors, with Crohn's disease for example increasing the risk of SBA [8]. Patients with familial adenomatous polyposis (FAP), hereditary non-polyposis colorectal cancer (HNPCC), Peutz-Jeghers syndrome, and celiac disease also show an increased risk of SBA development $[9,10]$. To our knowledge, this is the first report of a patient with simultaneous multiple synchronous SBA and pulmonary tuberculosis. This is a rare case. The patient had both a rare tumor and an infectious disease. This complex condition made it easier for non-specialist physicians to perform an incorrect diagnosis. This misdiagnosis reminds us that: Firstly, we should not ignore multiple synchronous SBA when diagnosing intestinal diseases (especially IBS). Secondly, intestinal tuberculosis may represent risk factor for multiple SBA, although the pathology does not provide sufficient evidence.

To date, no specific methods exist for the diagnosis of multiple synchronous SBA. Examinations must be completed and we should obtain the pathology of high-risk patients as much as possible. An interesting issue from this case was how to identify SBA among at-risk patients. Crohn's disease is typically accompanied by perianal abscesses, perianal fistula and extra intestinal manifestations. Intestinal tuberculosis presents as by pulmonary tuberculosis. Abdominal CT provides only limited diagnostic benefits and small bowel angiography is unsuitable for bowel obstructions. Multidetector row helical computed tomography [11], and magnetic resonance imaging [12] can facilitate the diagnosis of small bowel disease. Gastroscopy and colonoscopy should be performed, if the tumor is located close to the proximal duodenum or far from the terminal ileum. The remainder of the small bowel cannot be accessed without the use of video capsule endoscopy (CE) or double balloon 
enteroscopy (DBE). The definitive diagnostic yield of CE is as low as $20-30 \%$, whilst DBE accounts for $60-70 \%$ of the diagnostic yield for intestinal disease [13]. However, $\mathrm{CE}$ is suitable for diagnosing scattered, small, or multiple lesions, in addition to active bleeding. The procedure is convenient, non-invasive, safe and comfortable. Doctors should strive to obtain pathology under endoscopy and avoid diagnostic treatments and treatment delays. In addition, the levels of $\mathrm{Ca} 211$ and other tumor markers including Ca125, Ca153 progressively increased in this case. For high-risk factors, such as FAP, elevated tumor markers and abnormal imaging examinations, laparotomy is recommended.

To-date, surgical resection remains the major treatment for patients with SBA. The 5-year survival is poor and dependent on tumor stage: $50-60 \%$ for stage I, 39$55 \%$ for stage II, $10-40 \%$ for stage III and $3-5 \%$ for stage IV [14]. The majority of diagnosis of SBA occurs at stage IV. This is because the disease is easily misdiagnosed and surgery is delayed. No dedicated TNM staging for multiple synchronous SBA exists. A higher number of adjuvant therapies are used for SBA treatment due to its poor prognosis and high risk of relapse $[15,16]$. Chemotherapy is the main adjuvant strategy in patients with SBA. However, detailed chemotherapeutic regimens for SBA are lacking. The most common chemotherapy drug is fluorouracil but its benefits are modest. The patient had brain metastasis after 4 cycles of chemotherapy. Only the early diagnosis of multiple synchronous SBA with complete excision can improve long-term survival.

In this study, we highlighted how multiple synchronous SBA is rare and easily misdiagnosed during the early stages. In suspected cases, patients should receive complete tumor marker examinations, gastroscopy, enteroscopy or capsule endoscopy, and pathology under endoscopy. Active laparotomy should also be performed.

\section{Abbreviations \\ SBA: Small bowel adenocarcinoma; CT: Computed Tomography; T- spot: Mycobacterium tuberculosis specific T lymphocyte; TB-IGRA: Interferon- gamma release assay for Mycobacterium tuberculosis; ESR: Erythrocyte sedimentation rate; ASO: Anti-streptolysin; RF: Rheumatoid factor; Ca125: Carbohydrate Antigen 125; Ca153: Carbohydrate Antigen 153; Ca211: Carbohydrate Antigen 211; SCC: Squamous cell carcinoma antigen; FAP: Familial adenomatous polyposis; HNPCC: Hereditary non-polyposis colo- rectal cancer; CE: Capsule endoscopy; DBE: Double balloon enteroscopy; AFP: Alpha fetoprotein; MPO: Mouse myeloperoxidase; LCA: Leukocyte common antigen; TTF-1: Thyroid transcription factor-1; HER-2: Human epidermalgrowth factor receptor-2; CK: Cytokeratin; CgA: Chromogranin A; IBS: Inflammatory bowel disease}

\section{Acknowledgements}

Not applicable.

\section{Authors' contributions}

FJ and LY were responsible for the coordination of the project and contributed to the study design. The writing team consisted of QWL and TC. HXC, XX, CQC, and ZYS collected medical history data and followed up the patient. All authors read and approved the final manuscript.

\section{Funding}

No funding was received.

Availability of data and materials

All data and materials are available in this article.

Ethics approval and consent to participate

Not applicable.

\section{Consent for publication}

We obtained the patient's written consent for his personal and clinical details along with identifying images to be published in this study.

\section{Competing interests}

The authors declare no competing interests.

\section{Author details}

${ }^{1}$ Department of General Surgery, South Campus, Renji Hospital, School of Medicine, Shanghai Jiao Tong University, Shanghai 200112, China.

${ }^{2}$ Department of Nursing, South Campus, Renji Hospital, School of Medicine Shanghai Jiao Tong University, Shanghai 200112, China. ${ }^{3}$ Department of Tuberculosis Control, Shanghai Municipal Center for Disease Control and Prevention, Shanghai 200336, China. ${ }^{4}$ Department of Abdominal Surgery, Shanghai Tenth People's Hospital, Tongji University School of Medicine, Shanghai 200072, China. ${ }^{5}$ Department of Gastrointestinal Surgery, Renji Hospital, School of Medicine, Shanghai Jiao Tong University, Shanghai 200127, China

Received: 29 September 2019 Accepted: 7 April 2020

Published online: 16 April 2020

\section{References}

1. Frost DB, Mercado PD, Tyrell JS. Small bowel cancer: a 30-year review. Ann Surg Oncol. 1994;1(4):290-5.

2. Pan SY, Morrison H. Epidemiology of cancer of the small intestine. World J Gastrointest Oncol. 2011;3(3):33-42.

3. Halfdanarson TR, McWilliams RR, Donohue JH, Quevedo JF. A singleinstitution experience with 491 cases of small bowel adenocarcinoma. Am J Surg. 2010;199(6):797-803.

4. Dabaja BS, Suki D, Pro B, Bonnen M, Ajani J. Adenocarcinoma of the small bowel: presentation, prognostic factors, and outcome of 217 patients. Cancer. 2004;101(3):518-26.

5. Talamonti MS, Goetz LH, Rao S, Joehl RJ. Primary cancers of the small bowel: analysis of prognostic factors and results of surgical management. Arch Surg. 2002;137(5):564-70 discussion 570-561.

6. Cavalla C, Oppliger F, Schiappacasse G, Valderrama R, Castiblanco A, Margarit S. Multiple synchronous adenocarcinomas of the small bowel in a young patient: a case report. Int J Surg Case Rep. 2018;42:250-3.

7. Dasari BV, Gardiner KR. Management of adenocarcinoma of the small intestine. Gastrointest Cancer Res. 2009:3(3):121-2.

8. Gardiner KR, Dasari BV. Operative management of small bowel Crohn's disease. Surg Clin North Am. 2007;87(3):587-610.

9. Bennett CM, Coleman HG, Veal PG, Cantwell MM, Lau CC, Murray LJ. Lifestyle factors and small intestine adenocarcinoma risk: a systematic review and meta-analysis. Cancer Epidemiol. 2015;39(3):265-73.

10. Cahill C, Gordon PH, Petrucci A, Boutros M. Small bowel adenocarcinoma and Crohn's disease: any further ahead than 50 years ago? World J Gastroenterol. 2014;20(33):11486-95.

11. Boudiaf M, Jaff A, Soyer P, Bouhnik Y, Hamzi L, Rymer R. Small-bowel diseases: prospective evaluation of multi-detector row helical CT enteroclysis in 107 consecutive patients. Radiology. 2004;233(2):338-44.

12. Masselli G, Polettini E, Casciani E, Bertini L, Vecchioli A, Gualdi G. Smallbowel neoplasms: prospective evaluation of MR enteroclysis. Radiology. 2009:251(3):743-50.

13. Zhang ZH, Qiu CH, Li Y. Different roles of capsule endoscopy and doubleballoon enteroscopy in obscure small intestinal diseases. World J Gastroenterol. 2015;21(23):7297-304.

14. Aparicio T, Zaanan A, Mary F, Afchain P, Manfredi S, Evans TR. Small Bowel Adenocarcinoma. Gastroenterol Clin N Am. 2016;45(3):447-57. 
15. Shenoy S. Primary small-bowel malignancy: update in tumor biology, markers, and management strategies. J Gastrointest Cancer. 2014;45(4):421-30.

16. Han SL, Cheng J, Zhou HZ, Guo SC, Jia ZR, Wang PF. Surgically treated primary malignant tumor of small bowel: a clinical analysis. World J Gastroenterol. 2010;16(12):1527-32.

\section{Publisher's Note}

Springer Nature remains neutral with regard to jurisdictional claims in published maps and institutional affiliations.

Ready to submit your research? Choose BMC and benefit from:

- fast, convenient online submission

- thorough peer review by experienced researchers in your field

- rapid publication on acceptance

- support for research data, including large and complex data types

- gold Open Access which fosters wider collaboration and increased citations

- maximum visibility for your research: over $100 \mathrm{M}$ website views per year

At BMC, research is always in progress.

Learn more biomedcentral.com/submissions 